\title{
Semantic Web Engineering: Boon or Bane
}

Kartik Goel ${ }^{1}$, Ria Rawal ${ }^{2}$

${ }^{1}$ Bhagwan Parshuram Institute of Technology, GGSIPU, New Delhi, India ${ }^{2}$ Bhagwan Parshuram Institute of Technology, GGSIPU, New Delhi, India

\author{
kkartikgoel1999@gmaill.com ${ }^{2}$ riarawal99@gmail.com
}

\begin{abstract}
Semantic is the study of meaning of words in a sentence. Semantics help us find words which will fit better in sentence without changing the actual meaning of the sentence. Semantic is very important because, it is necessary to understand the different meanings of words and solves the problem of data overloading while retrieving information from Web. But semantics in ontological world has some shortcomings also. All the advantages and disadvantages related with semantics are collected and explicitly listed out clearly in this paper after a lot of research. Further, graphs have been used to depict the aim of the paper in a coherent manner and visualize the information written focusing on every aspect and need. The study of ontology and semantics in today's world is considered necessity and ontological axioms have found their use in many fields like medicine, laboratories, mathematical calculation etc. Through this paper, we propose to find if semantic web engineering is a boon or a bane in the field of technology.
\end{abstract}

Keywords - Semantic Web Engineering, WWW, description logic, Ontology.

\section{Introduction}

Semantic Web Engineering is the study which corroborates and controls rules to store and manipulate the data when needed. The data is collected and is enriched with an appropriate meaning so that it is well comprehended, without facing any sorts of difficulties. This paper lists out the advantages and disadvantages of this technology in order to determine if it is boon or bane for the future.

The main aim of this research paper is to provide a stand whether the Semantic Web is boon or bane by carefully analysing the advantages and various disadvantages it offers.

This field has been becoming more popular recently and has been proved beneficial in many domains. This is so because it helped the researchers and experts in the field of Computer Science to overcome the problem of information or excessive data overload with the growth in size of WWW. This has made the Web accessible to everyone across the globe and has made it easier to share and transfer information.

Semantic Web focuses on the meaning and not just the structure or syntax. It is highly enriched with various number of Web languages such as RDF [1], DAML+OIL [2], RDFS [3], OIL [4] and not just HTML, in order to hasten the process of retrieving the correct data. In Fig (1) one can observe the basic structure of the Semantic Web which lies on the bed of XML based upon a character set used for encoding and certain identifiers. It also incorporates the study of Ontology [5] which uses a certain logic in order to prove the validity of certain axioms made in Description Logic [6]. Description logic [7] then further uses a methodology of reasoning in order to understand the knowledge base available in the form of natural language by translating axioms to an ontological feature diagram [8] and data model and verifying the relationship among different roles, concepts and other entities. Individual names of classes are represented via constants which are used to signify a particular individual whereas concepts signify larger set of individuals. Relationships or links created between them are termed as roles. In the architecture or the structure of Semantic Web there exists a unit of SPARQL or even CSPARQL [9] that plays a pivotal role in providing different queries.

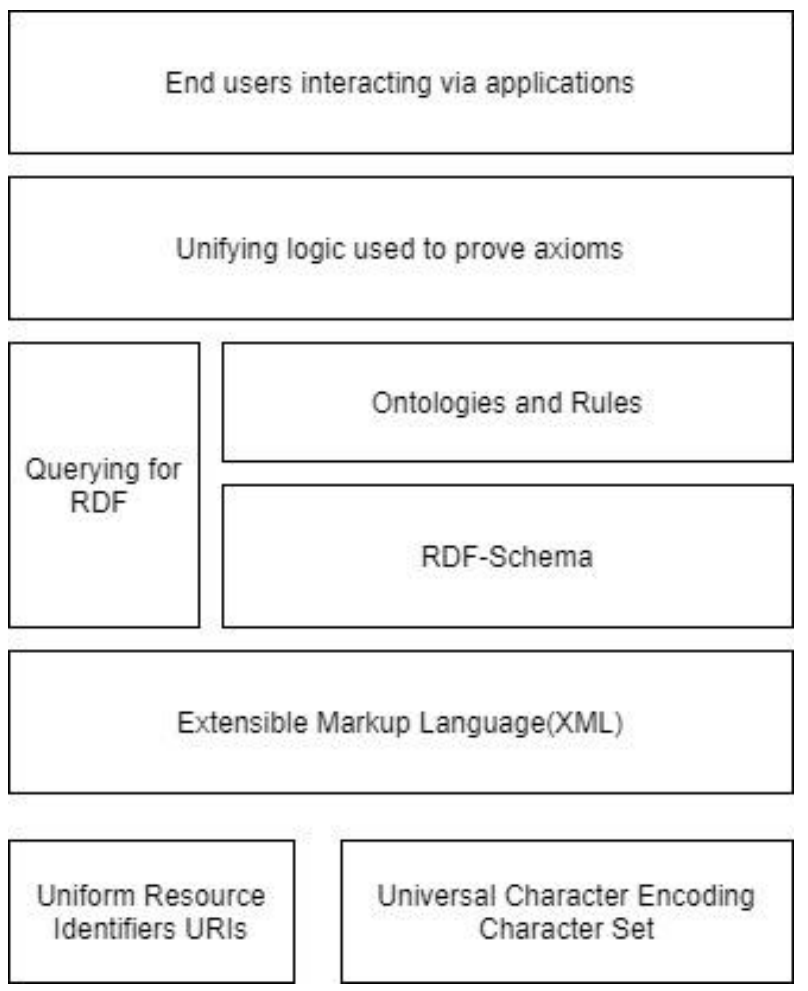

Fig.1 Basic Structure of Semantic Web

Despite having such an enriched web language structure with many advantages there still exist certain drawbacks which 
makes it an important field of research and extensive study. The quality of the existing structure of Semantic Web can be enhanced by working more and advancing the technology in a three-dimensional aspect. The paper is organized into the following sections: Section 2 explains and lists out the advantages of Semantic web, Section 3 explains and lists out its disadvantages and at last the paper is unambiguously concluded which is then followed by a list of references.

\section{Semantic Web Engineering: Boon}

Recently over the past few years the use of ontological data modelling and semantic web engineering have been burgeoning in the field of terminology and elsewhere. Thus, now this paper aims to examine and further briefly explicate the advantages that Semantics play which are listed as follows:

- Effective Communication: Semantics affects the communication in greater ways, no part of communication is complete without understanding and realizing the importance of semantical meaning in communication.

- Well Organized Language: Semantic is a wellorganized language. It validates facts and provides meaning to the given content. The entire meaning of the sentence gets a proper check and validation. This is done by checking the consistency of the ontology that is represented in description logic and which is then provided to a reasoner.

- Removal any sorts of Ambiguity: Semantic, also serves the purpose of removing structural ambiguity from text. Proper meaning and sentence formation don't allow ambiguity.

- Establishment of Relationship: The primary aim of semantics is to depict relationship among different words in a sentence (shown as concepts in the study of ontology) and control the meaning of sentences. Study of semantics make the statement of sentences true or false according to the situation.

- Identifies terms based on their Nature: Semantic ontology on the broader terms, involves meaning of words along with the relationship to one another. Semantic ontology helps us serve with two important purposes:

1. Helping in Identifying Common Terms: Words which possess same or similar meaning fall under this category. All synonyms of a given word fall under this category.

2.Helping in Identifying Different Terms: Words which possess different or opposite meanings to a selected word fall under this category. All antonyms of a given word fall under this category.

- Provide Suitable meanings: Semantic helps different parts of the sentence to interact with each other and form connection with one- another. Synonyms often easily replace the original word, but not every time.

1) Homonyms: Homonyms are also confusing in this sense, as they have more than one meaning for example book has two meaning, one is the collection of pages and other to make some kind of reservation.
2) Antonyms: Antonyms are words possessing opposite meanings. For example: sad is the antonym of the word 'happy' and can be used according to the meaning we need to give to the sentence. It helps us find suitable words for example in happy and joyous have similar meanings, but their usage in a sentence will depend on the kind of sentence, we want to form.

- $\quad$ Provides Reasoning of Facts: Semantic Ontologies help in building relationship between different concepts, allowing proper reasoning tools [10] to check the coherency about text or facts in a sentence

- Helps in formulation of Axioms: Semantic data collected from various sources also enable formation of ontological axioms, used for the purpose of verification of facts. Semantic data is stored in databases and referred to, whenever needed.

- Helps in Linking Process: Semantic reasoning feature help in linking concepts and domains. Semantic Ontology helps to shift from one concept to another. Semantics also helps in extending support to different processes and data structures, which need semantic information to proceed further.

- Easy plotting of Graphs: Semantic reasoning is done through semantic graph formation, which uses facts or information stored in databases for plotting of graphs. The information is fully verified information and thus, reliable. The information plotting is easy and one of the important advantage of semantics engineering.

- Lower amount of Efforts put into programming: The figure (2) shows a comparison depicting the amount of effort put into programming in different applications like Google Search Engine and CiteSeerX as compared to Semantic Web related Concepts. It is observed that CiteSeer takes a lot of time and effort in programming as it undergoes a strict scrutinization of the content present in the documents such as the references and citations, etc. On the other hand, Semantic has its knowledge base derived directly from the Web which makes it easier and faster to work and program accordingly.

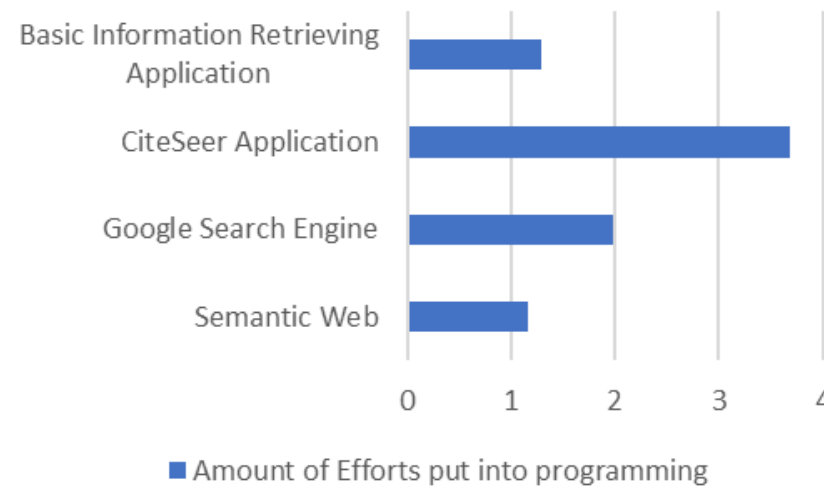

Fig.2 Comparison showing the amount of efforts put in programming in various standard web applications 
All these advantages have expanded the use of Semantic Web to a wider degree ranging from its applications in the field of medicine to performing certain tedious mathematical calculations and also in laboratories. This has been achieved by combining new technologies like Natural Language processing and concepts of Machine Learning with it which plays an efficacious role in data management (includes both structured and non-structured data).

\section{Semantic Web Engineering: Bane}

Even though Semantic Web is an emerging field which is proved to be advantageous in many aspects it does however possess certain drawbacks which cannot be ignored and should be taken into consideration while applying them in various fields. Certain effective shortcomings of this technology are listed as follows:

- Synonymous Representation of concepts in Ontologies: While ontology modelling or designing there can be an ambiguity that might arise due to two concept names having similar or synonymous meanings.

- Lacks effective Scalability: Scalability refers to the manner in which data is being stored and managed. So, there are some issues which makes it difficult to organize or to find the appropriate and correct Semantic web content.

- Lack of Knowledge: Semantic Web Engineering although an upcoming field still has many limitations which can only be overcome if more time and research is put into it. Thus, more trailblazing methods and research designs should be adopted in order to expand the horizons of this field and make it easily accessible and understandable to all in future.

- Limited availability of Semantic Web related Content: The Semantic Web content available presently is very sparse because it is a technology whose architecture is still in the process of construction which is based on many languages such as RDF, RDFS(RDF-Schema), DAML, etc. The comparison in the efforts required to prepare the Semantic Web content have been shown in figure(3). We It is very well observed from the graph that certain web applications like Google, CiteSeerX and other basic Information retrieval applications need quite less efforts as compared to the Semantic web in order to prepare the content. This can be because the concepts (following certain constraints) have to put in a greater amount of effort in order to translate the knowledge base into appropriate Semantic Web Content. Therefore, this could possibly be a major reason behind the limited availability of the Semantic Web related content.

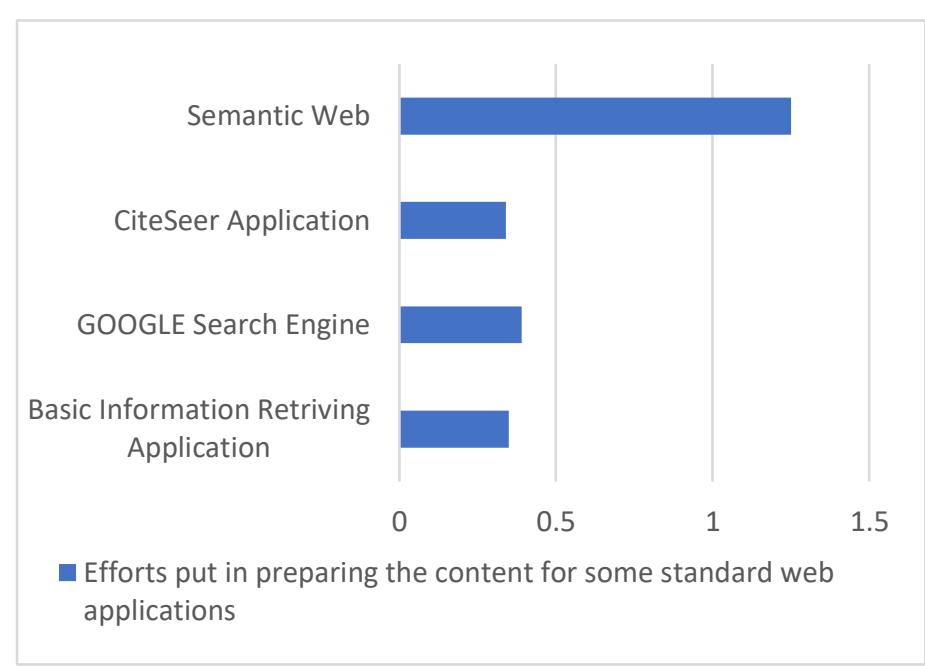

Fig 3. Comparison showing the amount of efforts put in preparing the content for various standard web based applications

- Lack of tools: Taking the present scenario in consideration then there are only two ways in which the users can build ontologies for a domain which are using:

1.Some customary editing tools used for building ontology like the Protégé which can be a tedious and an arduous process for engineers to use as it includes many difficult technicalities such as certain logical assumptions or inferences acquired from the knowledge base and it can be confusing if some features get ignored or missed out.

2. Or, by using Ontoterm which is a terminology management system used for building ontologies. It too possesses many restrictions and cannot handle multilingual data.

3.Also, better tools for checking consistency in various ontologies is still pending in thus field

- Difficulty in translating knowledge base into Description logic: This disadvantage is related to the difficult to translate a given knowledge base into its equivalent Description logic form (DL). Description Logic [11] is a language which is considered to be the backbone of ontology. It basically deals with representing the knowledge base into axioms [12] with the use of certain advanced constructors like conjunctions [13], equivalence classes, disjunctions, etc. Although the technical experts or ontology engineers may find it easy at first but there are many minute technicalities involved in between the process of translation. Not only this but many a times the data provided from certain sources is not well interpreted. This consequentially disrupts the relationship between the terminologists and the domain(technical) experts.

- It lacks an advanced mechanism which makes it difficult to find the correct content on Web [14]: The Semantic web consists of basic structure wherein pages are connected via hyperlinks. It lacks a mechanism to develop applications which can group together related pages and give some additional valuable services or extensions thereby 
saving time and also improving the extensibility of semantics by providing extensions as well as accurate data also.

- Visualization of Data: When the amount of data stored increases a certain limit or there in an overload of data then visualization plays a vital role in displaying the content. Thus, viewing the Semantic web content in a three-dimensional technique can make data visualization in Semantic more unique and easier to understand.[15]

A major drawback while creating an ontology (which plays a pivotal role in Semantic Web engineering and data modelling) is that certain synonyms are misunderstood and are represented as two different entities or concepts which increases the degree of ambiguity in the process of representing a knowledge base.

\section{Conclusion and Future Scope:}

In this paper various advantages and shortcomings of the Semantic Web have been aptly listed out and described. The field of Semantics offers to solve the problem of data overload, improves the efficiency of understanding by focusing on the meaning than syntax, also helping in formulating the sentences present in natural language to its easier equivalent axioms in description logic and further making it easier to program. But on the other hand, with many such major advantages comes certain disadvantages too. Some of the most important ones being the availability of content and the irksome process of preparing the content for it. Lack of tools and knowledge to handle them poses another major threat in the way of Semantic Web Engineering which must be dealt with at earliest.

In this paper the focus was on analysing the pros and cons of this technology in order to highlight the areas where research and extensive attention of experts is required. Thus, enhancing the visualization can improve the current state of Semantic Web architecture. With the advancement in existing web languages and tools in use this field could prove to be a boon in not just scientific and technical domains like industries of software production [16] but also in social and economic sectors. By overcoming these obstacles this paper provides the vision that Semantic Web Engineering would definitely prove to be a boon in future.

References:

[1] Lassila, O., Swick, R. Resource Description Framework (RDF) Model and Syntax Specification. W3C Recommendation. February, 1999.

http://www.w3.org/TR/REC-rdf-syntax/

[2] Reference description of the DAML+OIL language. http://www.daml.org/2001/03/reference.html Frank van Harmelen, Peter F. Patel-Schneider and Ian Horrocks, editors.

[3] Brickley, D., Guha, R.V. Resource Description Framework (RDF) Schema Specification. W3C Proposed Recommendation. March, 2000. http://www.w3.org/TR/rdfschema/

[4] Horrocks, I., Fensel, D., Harmelen, F., Decker, S., Erdmann, M, Klein, M. "OIL in a Nutshell". Proceedings of the 12th European Workshop on Knowledge Acquisition, Modeling, and Management (\{EKAW\}'00), R. Dieng
[5] Völker, J., Hitzler, P., \& Cimiano, P. (2007, June). Acquisition of OWL DL axioms from lexical resources. In European Semantic Web Conference (pp. 670-685). Springer, Berlin, Heidelberg Haarslev, V., \& Möller, R. (2000). Optimizing TBox and ABox Reasoning with Pseudo Models. In Description Logics (pp. 153-162).

[6] Eiter, T., Lukasiewicz, T., Schindlauer, R., \& Tompits, H. (2004, November). Well-founded semantics for description logic programs in the semantic web. In International Workshop on Rules and Rule Markup Languages for the Semantic Web (pp. 81-97). Springer, Berlin, Heidelberg.

[7] Hofmann, M. (2005, June). Proof-theoretic approach to description-logic. In 20th Annual IEEE Symposium on Logic in Computer Science (LICS'05) (pp. 229-237). IEEE.

[8] Czarnecki, K., Hwan, C., Kim, P., \& Kalleberg, K. T. (2006, August). Feature models are views on ontologies. In 10th International Software Product Line Conference (SPLC'06) (pp. 41-51). IEEE.

[9] Barbieri, D.F., Braga, D., Ceri, S., Della Valle, E., Grossniklaus, M.: C-SPARQL: SPARQL for continuous querying. In: Proceedings of the 18th International World Wide Web Conference (WWW 2009), Madrid (2009)

[10] Koutsomitropoulos, D., Solomou, G., Pomonis, T., Aggelopoulos, P., \& Papatheodorou, T. (2010, April). Developing distributed reasoning-based applications for the Semantic Web. In 2010 IEEE 24th International Conference on Advanced Information Networking and Applications Workshops (pp. 593-598). IEEE.

[11] Hakim, M. M., \& Garrett, J. H. (1993). A description logic approach for representing engineering design standards. Engineering with Computers, 9(2), 108-124.

[12] Vasilecas, O., Kalibatiene, D., \& Guizzardi, G. (2009). Towards a formal method for the transformation of ontology axioms to application domain rules. Information Technology and Control, 38(4).

[13] Gilio, A., \& Sanfilippo, G. (2013). Conjunction, disjunction, and iterated conditioning of conditional events. In Synergies of soft computing and statistics for intelligent data analysis (pp. 399-407). Springer, Berlin, Heidelberg

[14] Osborne, F., Motta, E., Mulholland, P.: Exploring scholarly data with rexplore. In: Alani, H., et al. (eds.) ISWC 2013, Part I. LNCS, vol. 8218, pp. 460-477. Springer, Heidelberg (2013).

[15] Frank van Harmelen, Jeen Broekstra, Christiaan Fluit, Herko ter Horst, Arjohn Kampman, Jos van der Meer and Marta Sabou, "Ontology-based Information Visualisation", Proceedings of the workshop on Visualisation in conjunction with the 5th International Conference on Information Visualisation, London 2001

[16] Vangipuram Radhakrishna, C.Srinivas, (2013)"Document Clustering Using Hibrid XOR Similarity Function For Efficient Software Component Reuse", Procedia Computer Science, Vol.17,pp.121-128. 